\title{
THE $N(S)$ RELATIONSHIP AT 1400 MHz
}

\author{
A. H. BRIDLE \\ Queen's University, Kingston, Ontario, Canada \\ and \\ M. M. DAVIS \\ National Radio Astronomy Observatory, ${ }^{*}$ Green Bank, W. Va., U.S.A.
}

\begin{abstract}
The number-flux density relation for radio sources selected at $1400 \mathrm{MHz}$ is discussed, the exponent of the counts being given for two flux density ranges. The accuracy of the exponents is limited only by the statistical size of the sample. The completeness of the catalogue is estimated. Results are compared with other flux density measurements.
\end{abstract}

The parameters of the number-flux density relationship determined from two observing programs with the NRAO 300 -foot telescope are shown in Table I. The exponent of the integral number counts at $1400 \mathrm{MHz}$ for sources at $|b| \geqslant 20^{\circ}$ is 1.90 to a limit of 2.0 f.u. ${ }^{* *}$, and 1.50 between 2.0 and 0.5 f.u. The following features of these results should be noted.

\section{TABLE I}

Areal density of radio sources at $1400 \mathrm{MHz}$

$|b| \geqslant 20^{\circ}$, equivalent diameters $<10$ arc $\min$

\begin{tabular}{ll} 
Flux density limit & $N /$ Steradian \\
\hline$S \geqslant 2.0$ f.u. & $54.5 \pm 3.6$ \\
$S \geqslant 0.5$ f.u. & $442 \pm 31$ \\
\hline Flux density range & Integral slope \\
\hline$S \geqslant 2.0$ & $1.90 \pm 0.13$ \\
$0.5 \leqslant S<2.0$ & $1.50 \pm 0.08$
\end{tabular}

(1) The precision of the flux densities of the individual sources, $\pm(2 \%+0.03$ f.u. $)$, is considerably greater than that available at lower frequencies. Our results therefore demonstrate that the high value of the exponent for intense sources is not due to observational errors. The results are consistent with the Cambridge 178 and $408 \mathrm{MHz}$ counts (Gower, 1966; Pooley and Ryle, 1968).

(2) For the intense sources the statistical counting (sample-size) errors, determined from the differential counts by the method of Crawford et al. (1970) exceed those due to noise and confusion, determined by Monte Carlo trials, by a factor of order 10 . The uncertainty of 0.13 in the exponent of the number counts for the intense sources is therefore determined entirely by the size of the sample at $|b| \geqslant 20^{\circ}$ obtained by

* Operated by Associated Universities, Inc., under contract with the National Science Foundation.

** 1 f.u. $=10^{-26} \mathrm{Wm}^{-2} \mathrm{~Hz}^{-1}$. 
counting approximately one hemisphere. Extension of this work to the southern sky would still leave an uncertainty of at least 0.1 in the exponent, which could not be further reduced.

(3) The results given in Table I suggest that the number count at $1400 \mathrm{MHz}$ cannot be represented by a simple power law to a limit of $0.5 \mathrm{f}$.u. The choice of flux density ranges for which the best-fitting exponents are given is, however, one of convenience, and should not be interpreted as indicating a discontinuity in the exponent near $2 \mathrm{f}$.u.

(4) The counts refer to sources with equivalent gaussian diameters up to 10 arc min, and use integrated flux densities. A trial using peak rather than integrated flux densities did not change the exponent of the counts significantly, due to the small number of sources with equivalent diameters between 3 arc min and 10 arc min.

The results shown in Table I come from (1) an intense source catalogue containing 234 sources $\geqslant 2.0 \mathrm{f}$.u. in the 4.30 ster area defined by $-5^{\circ}<\delta<70^{\circ},|b| \geqslant 20^{\circ}$, and (2) a full survey containing 200 sources $\geqslant 0.5 \mathrm{f}$.u. in the 0.45 ster area defined by $23^{\circ} 30^{\prime}<\delta<30^{\circ} 30^{\prime},|b| \geqslant 20^{\circ}$. The full survey is complete, and the intense source catalogue is $98 \%$ complete. All data were taken with $150 \mathrm{~K}$ system temperature, $60 \mathrm{MHz}$ bandwidth, $1400 \mathrm{MHz}$ center frequency, and beamwidths of 10.3 arc min in right ascension and $11.1 \mathrm{arc} \min$ in declination. The $E$-vector was in position angle $0^{\circ}$.

The intense source catalogue was compiled by reobserving sources from $1400 \mathrm{MHz}$

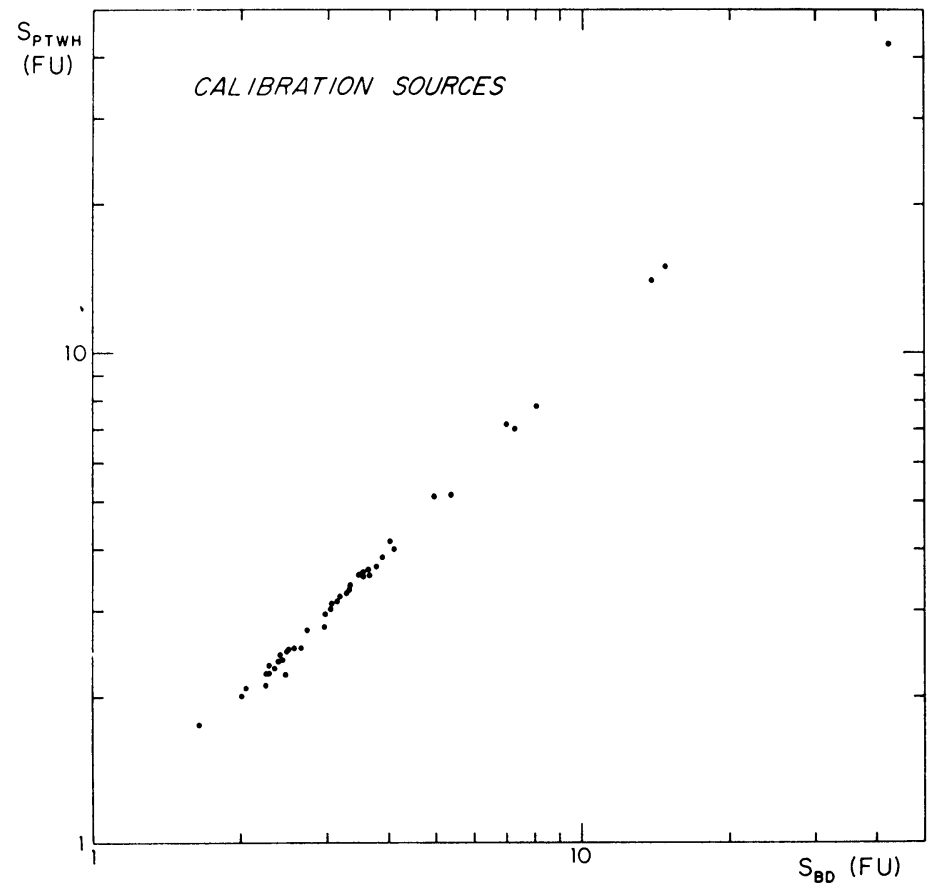

Fig. 1. Comparison of flux densities of sources measured by Pauliny-Toth et al. (1966) and by us which were used to interrelate these observations. Both sets of data have been corrected to the KPW flux-density scale (Kellermann et al., 1969). 
surveys made with smaller instruments. In each case an area of at least 40 arc min by 60 arc min centered on the quoted source position was mapped. This reobservation with the 300-ft telescope was necessary in order to provide reliable flux densities, and to reduce the effects of confusion, which proved to be large in some of the surveys (cf. Figures 2 and 3).

Flux densities of 44 isolated point sources measured by Pauliny-Toth, Wade and Heeschen (1966, henceforth referred to as PTWH) and corrected to the KPW flux density scale (Kellermann et al., 1969), have been used to normalize both the intense source catalogue and the full survey (cf. Figure 1). The ratios of the corrected PTWH flux density measurements to our present measurements show an rms scatter of $3.1 \%$ about the mean of unity.

The intense source catalogue includes the entire area of sky at $|b|>5^{\circ}$ searched by Galt and Kennedy (1968) with the Penticton 85-ft telescope (the DA survey). Sixty-two percent of this area has also been covered by at least one other $1400 \mathrm{MHz}$ survey (Kraus, 1964; Kellermann and Read, 1965; Kraus and Dixon, 1965; Kraus et al., 1966; Davis, 1967; Scheer and Kraus, 1967; Höglund, 1967; Dixon and Kraus, 1968; Fitch et al., 1969; Ehman et al., 1970). We have reobserved every source with $S_{1400} \geqslant 1.5 \mathrm{f}$.u. detected in these surveys for which adequate $300-\mathrm{ft}$ data were not already available in PTWH. Within the DA survey and for sources with unusually large flux density uncertainties, the reobservation was extended to sources with $S_{1400} \geqslant 1 \mathrm{f}$.u. We also reobserved sources which were excluded from the published DA survey because they had not previously been catalogued and had low signal-tonoise ratios (Galt and Kennedy, private communication).

These criteria ensure that membership of the final catalogue is not prejudiced by random or systematic errors in the flux densities given by the $1400 \mathrm{MHz}$ surveys used as finding lists.

\section{TABLE II}

Completeness of the intense source catalogue $-5^{\circ}<\delta<70^{\circ},|b|>20^{\circ}$, equivalent diameter $<10$ arc min

\begin{tabular}{lll}
\hline & $\begin{array}{l}\text { Number of } \\
\text { sources }\end{array}$ & $\begin{array}{l}\text { Area of sky } \\
\text { (steradians) }\end{array}$ \\
Penticton Survey & 210 & 4.30 \\
Other 1400 MHz Surveys & 18 & 2.68 \\
Other 1400 MHz Data & 7 & 1.62 \\
\hline & Completeness & Estimated number of \\
& & sources missing \\
\hline Penticton Survey & $88 \pm 3 \%$ & $29 \pm 3$ \\
BD Intense Source Catalogue & $98 \pm 2 \%$ & $4 \pm 3$ \\
\hline
\end{tabular}

Table II summarizes the completeness level of the intense source catalogue. It is essentially complete in the 2.68 ster area in which two or more independent surveys have been made. Three of the sources in this area were detected only in the DA survey; eighteen of the sources found in this area were not detected in the DA survey. 
The DA survey, when combined with the unpublished list mentioned above, is inferred to have a completeness of $88 \pm 3 \%$ at $2 \mathrm{f}$.u., from which we estimate that $11 \pm 3$ sources remain undetected at $1400 \mathrm{MHz}$ in the 1.62 ster area (primarily $30^{\circ}<\delta<70^{\circ}$ ) covered only by the DA survey. We have detected seven sources with $S_{1400} \geqslant 2.0$ by reobserving all sources not detected in $1400 \mathrm{MHz}$ surveys but which have published $1400 \mathrm{MHz}$ flux densities $\geqslant 1.5 \mathrm{f}$.u. These seven sources all lie in the region covered only by the DA survey. The remaining incompleteness of $4 \pm 3$ sources may represent a slight bias against flat-spectrum sources, as the seven sources added all come from low-frequency catalogues. However, the $2 \pm 2 \%$ incompleteness introduces a much smaller error in determining the number-flux density relation than does the statistical error due to the limited size of the sample.

The present flux density observations may be used to compare the flux-density scales and random errors of the finding surveys. In Figure 2 the present measurements are compared with those of the DA and Ohio surveys, and with Parkes and PTWH

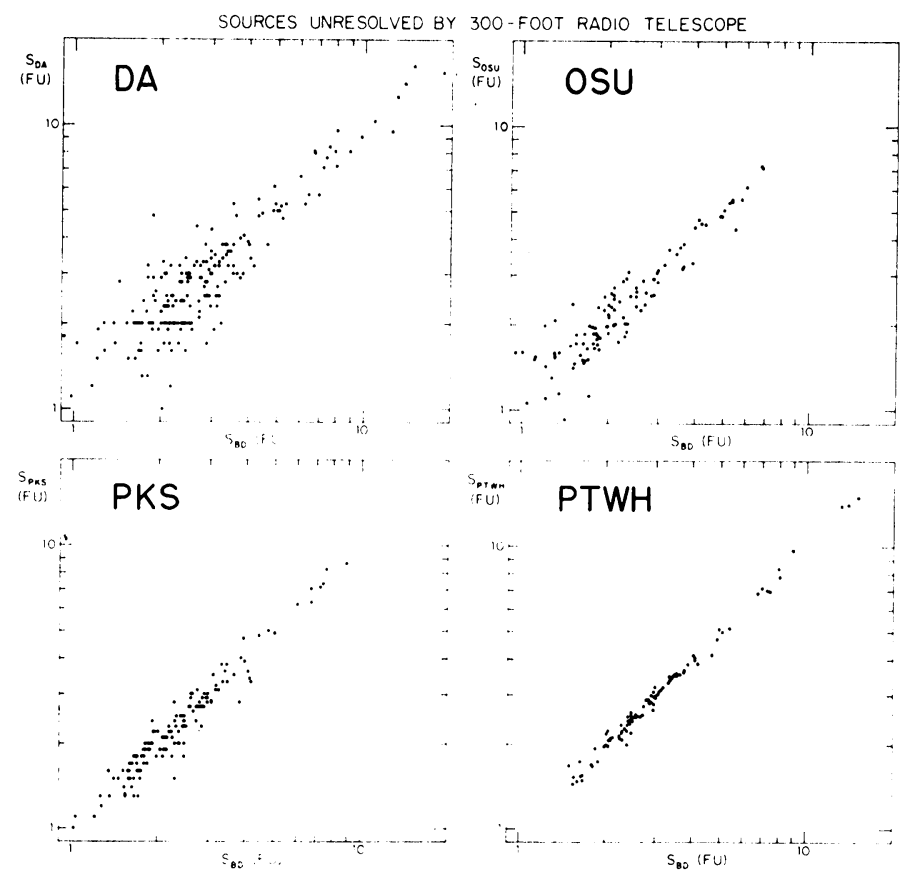

Fig. 2. Comparison of flux densities of unresolved sources measured with the 300-ft telescope in the present program $\left(S_{B D}\right)$ with values quoted in the DA survey, the Ohio State survey, the Parkes catalogue, and the NRAO catalogue.

measurements. A systematic over-estimation of flux densities relative to the KPW flux density scale is apparent in the Ohio survey (Figure 3); the effect is greater for sources noted as extended or confused in that survey.

The number count was extended to 0.5 f.u. using data from a full survey with the 
$300-\mathrm{ft}$ telescope between declinations $23^{\circ} 30^{\prime}$ and $30^{\circ} 30^{\prime}$. A sample area from the full survey is shown in Figure 4. The observing system was identical to that used for the intense source catalogue. Monte Carlo trials showed that the uncertainty in the areal
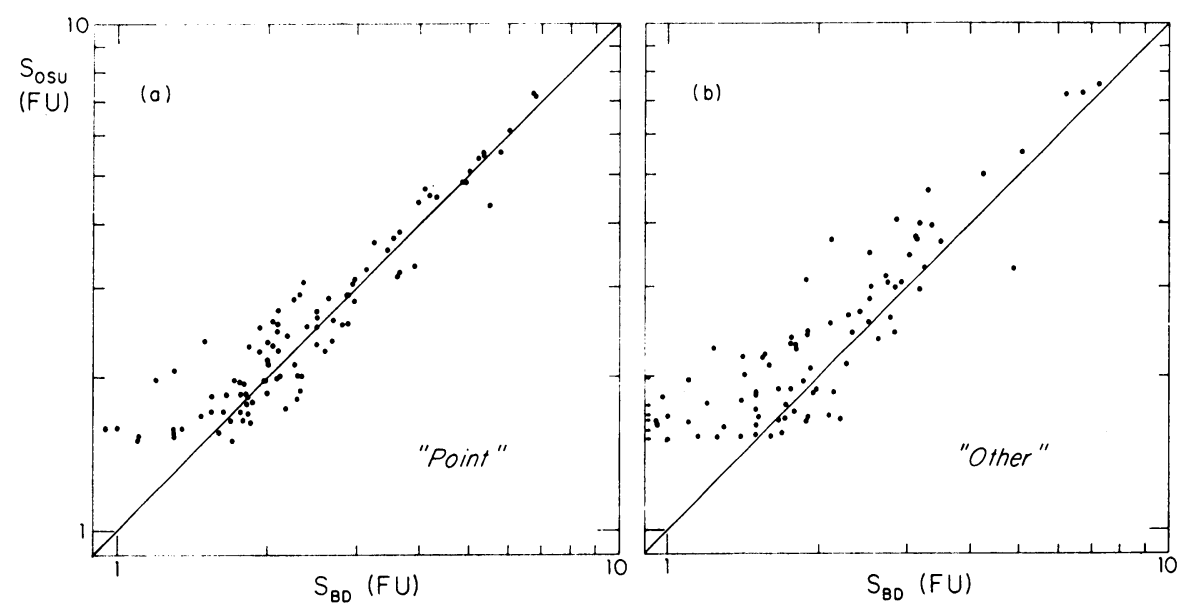

Fig. 3. Comparison of flux-densities $S_{B D}$ measured in the present program with flux-densities $S_{\mathrm{OSU}}$ from the Ohio survey for sources with $S$ osv $>1.5$ f.u. (a) Sources noted as 'point' sources in the Ohio catalogue. (b) Sources otherwise noted in the Ohio catalogue (extended, confused, unresolved group, etc.). The Ohio flux-densities for sources with $S_{B D}>2$ f.u. are systematically overestimated relative to the KPW flux-density scale, particularly for the non-'point' sources.

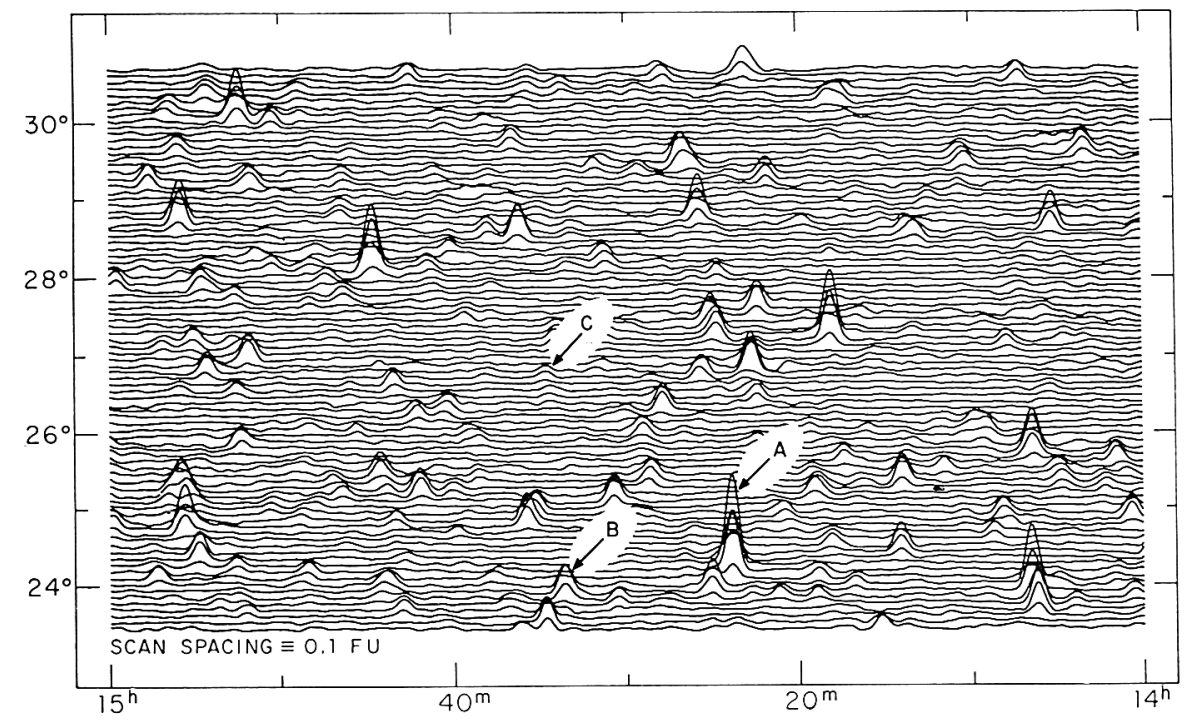

Fig. 4. Six percent of the $1400 \mathrm{MHz}$ full survey. The most intense source in the field (A) has a flux density of 1.59 f.u. The faintest source counted (B) has a flux density of 0.50 f.u. The faintest distinguishable objects have flux densities of about $0.1 \mathrm{f}$.u. (C) Fifteen of the 200 sources counted lie in this field. 


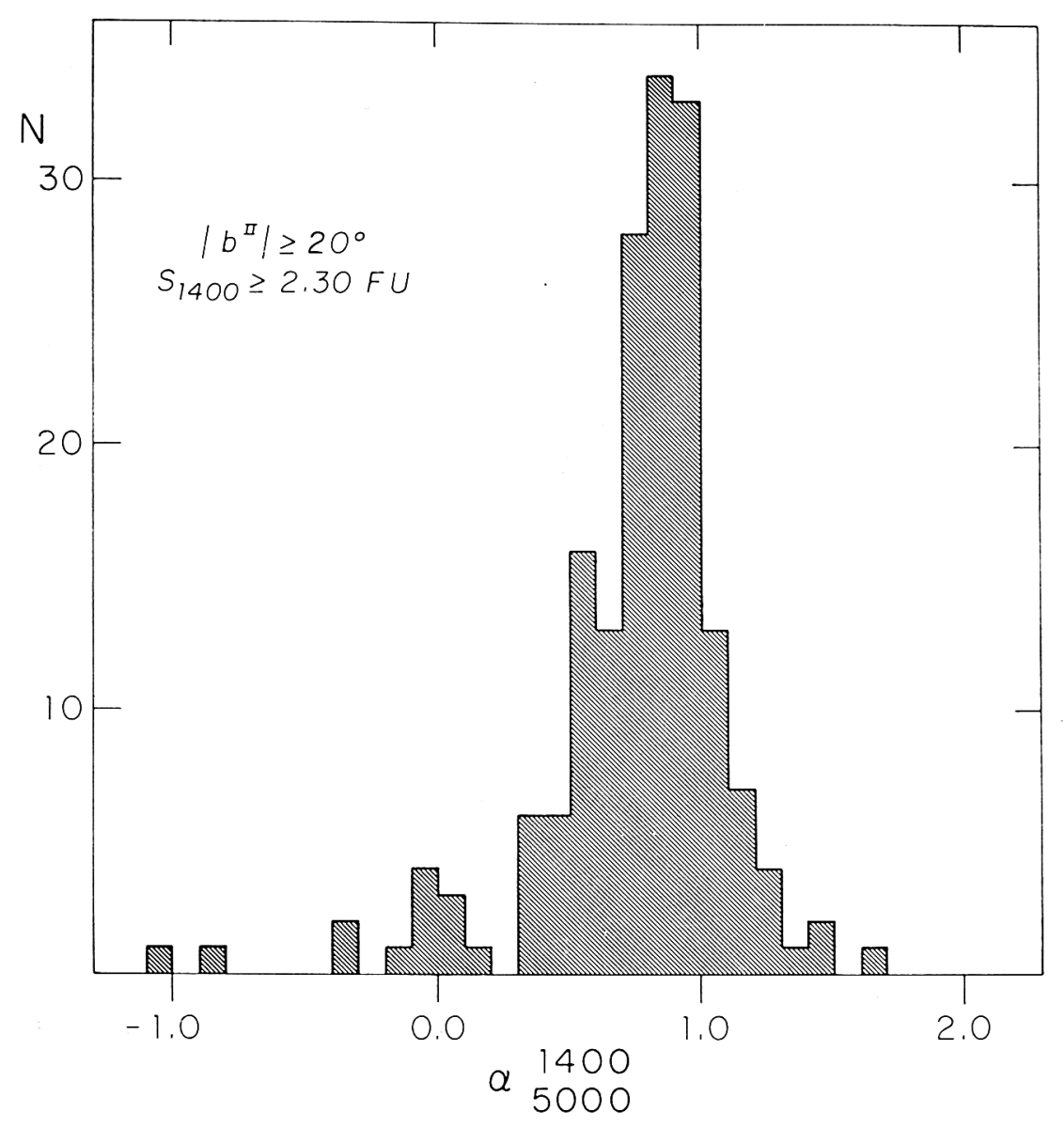

Fig. 5. The spectral index distribution for 177 of the 184 sources in the intense source catalogue with $S_{1400} \geqslant 2.30$ f.u.

density at $0.5 \mathrm{f}$.u. arising from the effects of noise and confusion is negligible compared with that due to the limited size of the sample.

The distribution of the spectral index $\alpha_{1400}^{5000}\left(S \propto v^{-\alpha}\right)$ shown in Figure 5 has been determined from data on 177 of the 184 sources in the intense source catalogue having $S_{1400} \geqslant 2.3$ f.u. Those sources not measured by Pauliny-Toth and Kellermann (1968) or by Shimmins et al. (1969) have been observed with the Algonquin Park 150-ft telescope at 3240, 6630 and $10630 \mathrm{MHz}$ (Bridle et al., in preparation), and the spectra have been interpolated to $5000 \mathrm{MHz}$. The fraction of sources with $\alpha_{1400}^{5000}<0.5$ is $16 \pm 5 \%$.

\section{References}

Crawford, D. F., Jauncey, D. L., and Murdoch, H. S.: 1970, Astrophys. J. 162, 405.

Davis, M. M.: 1967, Bull. Astron. Inst. Neth. 19, 201.

Dixon, R. S. and Kraus, J. D.: 1968, Astron. J. 73, 381. 
Ehman, J. R., Dixon, R. S., and Kraus, J. D.: 1970, Astron. J. 75, 351.

Fitch, L. T., Dixon, R. S., and Kraus, J. D.: 1969, Astron. J. 74, 612.

Galt, J. A. and Kennedy, J. E. D.: 1968, Astron. J. 73, 135.

Gower, J. F. R.: 1966, Monthly Notices Roy. Astron. Soc. 133, 151.

Höglund, B.: 1967, Astrophys. J. Suppl. 15, 61.

Kellermann, K. I. and Read, R. B.: 1965, Publ. Owens Valley Radio Obs. 1, No. 2.

Kellermann, K. I., Pauliny-Toth, I. I. K., and Williams, P. J. S.: 1969, Astrophys. J. $157,1$.

Kraus, J. D.: 1964, Nature 202, 269.

Kraus, J. D. and Dixon, R. S.: 1965, Nature 207, 587.

Kraus, J. D., Dixon, R. S., and Fisher, R. O.: 1966, Astrophys. J. 144, 559.

Pauliny-Toth, I. I. K., Wade, C. M., and Heeschen, D. S.: 1966, Astrophys. J. Suppl. Ser. $13,65$.

Pauliny-Toth, I. I. K. and Kellermann, K. I.: 1968, Astron. J. 73, 953.

Pooley, G. G. and Ryle, M.: 1968, Monthly Notices Roy. Astron. Soc. 139, 515.

Scheer, D. J. and Kraus, J. D.: 1967, Astron. J. 72, 536.

Shimmins, A. J., Manchester, R. N., and Harris, Beverley J.: 1969, Australian J. Phys. Astrophys. Suppl. 8.

\section{Discussion}

Lequeux: Interferometric measurements of a large number of radio sources have been made at Owens Valley using the 2 element interferometer at $1420 \mathrm{MHz}$. When combining these measurements with those by Fomalont, east-west structure is available for about $93 \%$ of all sources with flux density larger than 2 f.u. at $1420 \mathrm{MHz}$, in the area $-5^{\circ}<\delta<+70^{\circ},\left|b^{\mathrm{II}}\right|>10^{\circ}$. A preliminary result from this study is that high brightness temperature sources seem to have a steeper $\log N / \log S$ relationship than have the low brightness ones; but this difference is not really significant, owing to statistical errors resulting from the small size of the sample ( 285 sources). 